\title{
The Bond That Breaks: Closeness and Honor Predict Morality-Related Aggression
}

\author{
Theresa M. Benavidez ${ }^{1}$ Adon L. Neria ${ }^{1}$ • Daniel N. Jones ${ }^{1}$
}

Published online: 1 March 2016

C) Springer International Publishing 2016

\begin{abstract}
Endorsement of a "culture of honor" contributes to the belief that family honor is tied to female obedience across a variety of moral values. Violations of these moral values may lead to aggression. Male participants in studies 1 and 3 filled out a measure of cultural honor and closeness to their present wife or partner. Participants with high levels of both closeness and honor were most aggressive toward a hypothetical moral violation. In study 2 , we randomly assigned men to bond or not with a female confederate who devalued his most important moral value. Participants were then given the opportunity to aggress against her in a supposedly unrelated study by choosing how much painful hot sauce she would be forced to drink. Once again, high levels of closeness and honor predicted the greatest levels of aggression. In sum, moral disagreements by women are met with increased aggression within the culture of honor, the closer an honor-endorsing male is to the woman.
\end{abstract}

Keywords Interpersonal closeness · Self-expansion · Culture of honor $\cdot$ Honor-related violence

In 2012, Gul Meena, a 12-year-old girl from Pakistan, was in an arranged marriage to an abusive husband who was four times her age. After fleeing her abuser, Gul Meena's brother-enraged by her decision to disobey a cultural convention-found her in Afghanistan and hit her 15 times with an axe. Left for dead, she survived and has since found the

Daniel N. Jones

dnjones3@utep.edu; jonesdn@gmail.com; http://

academics.utep.edu/Default.aspx?tabid=72785

1 Department of Psychology, University of Texas, 500 W. University Ave., El Paso, TX 79968, USA courage to discuss her experience with honor-related violence (Ross 2013). Examples of honor-related violence, such as this, are not uncommon (Chesler 2009). At first blush, honorrelated violence against one's kin seems to contradict evolutionary theory (e.g., Dawkins 2006). However, if honor and conformity to cultural mores are perceived as vital to the inclusive fitness of the family, then the evolutionary motivations behind these acts come into focus.

In evolutionary terms, harming a romantic partner or kin creates dropped costs. However, such costs are smaller for men than they are for women, given that women possess a higher minimum burden of reproduction due to gestation and lactation. This minimum burden is absent in men, leaving them more likely to engage in violent acts against partners and offspring (Daly and Wilson 1988). Further, because cultural evolution has shaped the landscape of adaptation (e.g., Sommers 2009), adhering to societal norms is important for individual survival. To the degree that honor and reputation are central to one's culture, acquiring and retaining resources require a good reputation. Thus, survival may depend on reputation (Sommers 2009). Men may therefore be willing to engage in harmful behaviors against romantic partners or kin as a signal to others demonstrating the seriousness with which they take their reputation (Cohen and Nisbett, 1994; Henrich 2009). We argue that the closer two people are, the stronger the instinctual drive one may have to lash out at the other for a morality-related transgression. This assertion stems from the fact that close relationships result in increased psychological overlap between self and close other (Aron and Aron 1986). In this way, the closer people are to their family or partner, the more those people "represent" their self-concept. Thus, moral transgressions would be especially hurtful and egregious when committed by close others, and may have increased relevance for one's reputation. Thus, we argue that honorfocused men who feel that their reputation is being threatened 
by the actions of a close other will act aggressively toward that person. Further, we argue that increases in closeness will increase the severity of this aggressive reaction.

In cultures emphasizing family honor, violence against women is often perceived as socially acceptable, and even expected, in response to perceived disobedience or sexual impurity (Sev'er and Yurdakul 2001). Although "honor" assumes different forms across the world (Leung and Cohen 2011), female chastity and obedience are commonly emphasized (Vandello and Cohen 2003). Among cultures of honor, female disobedience or sexual impurity are perceived as harmful to a family's reputation, the manhood of male relatives, as well as the honor and manhood of her husband (Cohen et al. 1996). For example, Vandello and Cohen (2003) found that men in a culture of honor, whose partners were unfaithful, were perceived as "less of a man" and could regain some lost manhood by acting aggressively toward her. Moreover, the woman was perceived by others as regaining some of her lost honor by accepting this abuse. Unlike most other types of domestic violence or crimes of passion, honor-regaining acts of violence are commonly premeditated (Sev'er and Yurdakul 2001). However, to date, research has not examined how closeness may moderate reactions to moral violations, especially against the backdrop of honor-related violence.

It may seem reasonable that interpersonal closeness to a female family member would be a protective factor against aggression. However, evolutionary research suggests that moral violations committed by women disproportionately affect the perceived honor of those to whom she is closest, thus giving them the most motivation to aggress against her (Daly et al. 1982). This is especially true if the woman engages in betrayal, such as infidelity, against a man (Daly and Wilson 1988). Buss and Duntley (2006) also argue that aggression evolved to solve a set of adaptive problems that have yet to be completely understood. Thus, close-other aggression may help solve an adaptive problem of morality-based group conflict by forcing uniform thinking when it comes to morality.

According to Haidt (2008), morality binds communities together in the service of common goals. However, these same moral foundations can blind individuals to the harm they may do in the service of these moral foundations (Graham et al. 2009). Further, having a community agree on a set of guiding principles pertaining to moral foundations means that disobedience can cause a loss of honor (Nisbett 1993). Thus, when a close other engages in behaviors (or even expresses thoughts) that threaten family honor, aggression is a way to maintain status or mitigate loss of honor (Nisbett 1993). In this way, within cultures of honor, maintaining societal status can be more central to evolutionary goals than the well-being of an individual within a close-knit group.

Evidence that closeness predicts increased aggression also comes from research demonstrating that an increase in oxytocin (a bonding hormone) is associated with increased anxiety and less forgiveness following a relational transgression (Tabak et al. 2011). Self-esteem is another factor in predicting aggression toward romantic partners (e.g., Anderson 2002). Buss and Duntley (2006) point out that disadvantaged heterosexual men, who often have low self-esteem, are more likely to aggress against a romantic partner in an attempt to keep her faithful. Although research is mixed as to the role that selfesteem plays in aggression in general (cf. Baumeister et al. 1996), in the case of honor and morality, low self-esteem may leave men even more vulnerable to reputational threats when compared with those high in self-esteem (Salmivalli 2001).

In sum, perceived needs to maintain family honor provokes aggression in some situations (Cohen et al. 1996). For those high in perceived cultural honor, female obedience and chastity are critical features in maintaining family honor (Sev'er and Yurdakul 2001). Further, when an honor-related violation occurs, violence is often used to restore social status of the family (Nisbett 1993). As Buss and Duntley (2006) point out, we have yet to explore the full variety of adaptive dilemmas that may provoke honor-related aggression. Given that morality is binding and blinding to a community, perceived disagreements with respect to morality may evoke aggression in close-others. Further, no research has examined (especially experimentally) the role of closeness on honor-related violence in response to moral violations. Therefore, the present studies examined whether even temporary bonding (known to cause self-other overlap; see Aron et al. 1992; Aron 2003; Aron et al. 1997) will increase honor-related aggression. Specifically, we predict that interpersonal closeness will increase aggression among honor-endorsing men from a culture of honor.

\section{Study 1—Exploring Self-Reported Aggression}

\section{Methods}

\section{Participants}

As an initial investigation into this question, a classroom convenience sample of 202 adult men ( 18 or older) was collected from a large southwestern university $\left(M_{a g e}=29.05\right.$, $S D=15.04 ; 83 \%$ identified as Hispanic or Latino, $7 \%$ identified as White, Caucasian or of European Decent, 10 \% identified as other ethnicities). The study was restricted to men because incidents of honor-related violence have primarily been perpetrated by men. Participants were told that they would be answering questions about their current romantic relationship or a hypothetical future spouse if they were not romantically involved. Of the 202 men, only the 92 who indicated that they were in committed relationships were included in the analyses. 


\section{Measures}

Honor We assessed individual endorsement of honor using the revenge subscale of the Culture of Honor Scale (COHS; Figueredo et al. 2004). We focused on the revenge subscale because it was most directly related to aggression. The COHS asks participants to read a series of scenarios involving two individuals: "John" and "Mary." In all scenarios, John or Mary were harmed or insulted by someone and either reacted aggressively or not. Participants then responded to these 16 brief scenarios, indicating their level of agreement with John's or Mary's reaction. High scores indicated high levels of individual sense of revenge-based honor. The COHS is scored on a Likert scale ranging from -3 (did much more than he/she should have) to +3 (did much less than he/she should have). The items were then averaged into a composite with acceptable internal consistency $($ Mean $=-1.04, S D=0.89, \alpha=.76)$.

Interpersonal Closeness In order to assess closeness to one's spouse, the Inclusion of Other in the Self Scale (IOSS; Aron et al. 1992) was used. The IOSS is a single-item indicator of closeness that uses seven pictures of overlapping circles to indicate how close one feels to a partner. For the present study, the circles were labeled 1 to 7 (Mean $=5.47, S D=1.73)$ ranging from least overlapping $(1=$ low closeness $)$ to most overlapping ( 7 =high closeness $)$.

Self-esteem In order to control for self-esteem, we used the first five items from Rosenberg's self-esteem inventory (Rosenberg 1965). Although previous research has suggested that even one item is sufficient to assess self-esteem (Robins et al. 2001), five items were used to compute internal consistency, which was acceptable $(\alpha=.79)$.

\section{Design and Procedure}

After filling out initial questionnaires, participants were asked to rank order ten moral values from 1 (most important) to 10 (least important): respecting authority, being loyal to loved ones, doing no harm to others, chastity (being sexually pure), being fair to others, helping others especially the poor, being faithful and devote to one's religion, being strong/tough in character, "turning the other cheek" when attacked, spreading good will to others.

Participants were then given the following instructions: "Think about your current (or future) wife. Imagine 1 day you find out that she violated the moral value you listed ABOVE AS NUMBER 1, with her behavior. Please indicate how you would react below." Participants were asked how likely they would be to engage in each behavior below on a scale of 1 (Not at all LIKELY) to 5 (Extremely LIKELY): hit her, threaten her, insult/scream at her, never speak to her again, kick her out, divorce her, give her love, and tell her family. The latter two items were included to obfuscate the purpose of our survey.

\section{Results and Discussion}

Because the present study is focused on overt and direct aggression, the following items were used to assess aggression: hit her, divorce her, kick her out, insult her, and never speak to her again (Mean $=1.86, S D=0.79, \alpha=.73)$, Threaten her was not used because its inclusion lowered the alpha reliability of the composite score. It was also necessary to control for the type of value participants endorsed. Specifically, individuals who endorsed a value such as doing no harm are less likely to be aggressive in response to its violation than are individuals who endorsed a value such as loyalty. Further, these values may interact with personal honor endorsement such that individuals, who are highly focused on values such as loyalty and high in personal honor endorsement, may be especially aggressive. To assess this, an indicator variable, "value type" was created $(-1=$ endorsing "doing no harm to others," "turning the other cheek," helping others," or "spreading good will;" 1 = endorsing "being strong/tough in character," "being faithful and devote to one's religion," "respecting Authority," "being loyal to loved ones," "chastity," or "being fair to others"). "Value type" and its interaction with honor were controlled in all analyses.

The intercorrelations among honor, value type, and closeness were all small and non-significant ( $p$ s $>.345)$. Variables were standardized and included in a two-step hierarchical linear regression. Step 1 included self-esteem, relationship length (in months), value type, honor, and closeness. Step 2 included honor $\times$ closeness and honor $\times$ value type interactions in Step 2. Self-esteem had no contribution to the model $(\beta=0.004$, $p=.983,95 \% C I=-0.39,0.40)$ and was not central to our primary focus pertaining to closeness and honor, thus selfesteem was dropped from the regression and will not be discussed further.

A final two-step hierarchical linear regression (see Table 1 for all regression results) included value type, honor, relationship length, and closeness in Step 1, and honor $\times$ closeness and honor $\times$ value type interactions in Step 2. The $R^{2}$ for the first step was .07, and for the full model, it was .11. However, neither Step 1 nor Step 2 was significant overall $(F<1.80$, $p>.10)$. Further, none of the variables were significant in Step 1: Honor $(\beta=0.002 ; 95 \% C I=-0.20,0.20 ; p=.98$ 2 ) closeness $(\beta=0.08,95 \% C I=-0.14,0.30 ; p=.736)$, value type $(\beta=0.10 ; 95 \% C I=-0.07,0.18 ; p=.896)$, although relationship length was marginal $(\beta=-0.21$; $95 \% C I=-0.43,0.002 ; p=.052)$. Further, there was no significant interaction $(\beta=0.11 ; 95 \% C I=-0.13,0.34 ; p$ $=.367$ ) between value type and honor. However, as predicted, there was a marginally significant interaction between 
Table 1 Regressions predicting aggression across three studies

\begin{tabular}{lccll}
\hline Independent variable & $\beta$ & $S E$ & $95 \% C I$ & $p$ \\
\hline Study $1(n=92)$ & & & & \\
$\quad$ Honor & -.01 & .11 & $-0.2,0.20$ & .914 \\
Closeness & .12 & .11 & $-0.10,0.33$ & .294 \\
Rel. length & -.20 & .07 & $-0.42,0.02$ & .070 \\
Value type & .08 & .11 & $-0.05,0.21$ & .205 \\
Honor $\times$ value & .11 & .12 & $-0.13,0.34$ & .367 \\
Honor $\times$ closeness & $.23^{\mathrm{a}}$ & .12 & $-0.002,0.47$ & .052 \\
Study 2 $(n=82)$ & & & & \\
Honor & -.07 & .69 & $-1.43,1.30$ & .921 \\
Bonding & .09 & .12 & $-0.16,0.32$ & .483 \\
Value type & .23 & .43 & $-0.62,1.08$ & .590 \\
Honor $\times$ value & .39 & .67 & -0.95 .1 .72 & .566 \\
Honor $\times$ bonding & $.24^{\mathrm{a}}$ & .12 & $.01,0.47$ & .045 \\
Study 3 $(n=87)$ & & & & .003 \\
Honor & $.36^{\mathrm{a}}$ & .12 & $0.13,0.60$ & .080 \\
Closeness & -.21 & .12 & $-0.45,0.03$ & .135 \\
Value type & -.19 & .13 & $-0.44,0.06$ & .011 \\
Honor $\times$ value & -.18 & .11 & $-0.44,0.08$ & .166 \\
Honor $\times$ closeness & $.29^{\mathrm{a}}$ & .13 & $0.07,0.51$ & .013 \\
\hline
\end{tabular}

${ }^{\mathrm{a}} p \leq .052$. Reported results are for step 2 of all regressions

honor and closeness $(\beta=0.22 ; 95 \% C I=-0.002,0.47 ; p$ $=.052$ ) such that high scores on both variables predicted highest levels of aggression. Figure 1 shows this interaction at one standard deviation above and below the mean. However, the results of a simple slopes analysis indicated that individual honor was not significantly associated with aggression at either one $S D$ above the mean of closeness $(B=.24$, $p=.103$ ) or at one $S D$ below the mean of closeness $(B=-.21, p=.223)$.

Though non-significant overall, the results of study 1 were useful in demonstrating how central interpersonal closeness was to predicting honor-based aggression. Relationship length was marginally significant in predicting aggression and that honor and closeness were only marginally significant when interacting with each other. It may stand to reason that the degree of closeness between two people may be more central in predicting honor-based aggression following a moral violation than originally thought. Thus, a subsequent laboratory study was conducted to more formally explore the relationship of interpersonal closeness in predicting honor-based aggression. The goals of the second study were to remove the extraneous noise of a convenience classroom sample, remove unnecessary predictors (namely self-esteem and relationship length), directly assess the impact of interpersonal closeness by manipulating it in a controlled laboratory setting, and introduce a behavioral measure of aggression (see Baumeister et al. 2007). In this follow up, the closeness that participants felt toward a moral-violating other would be experimentally

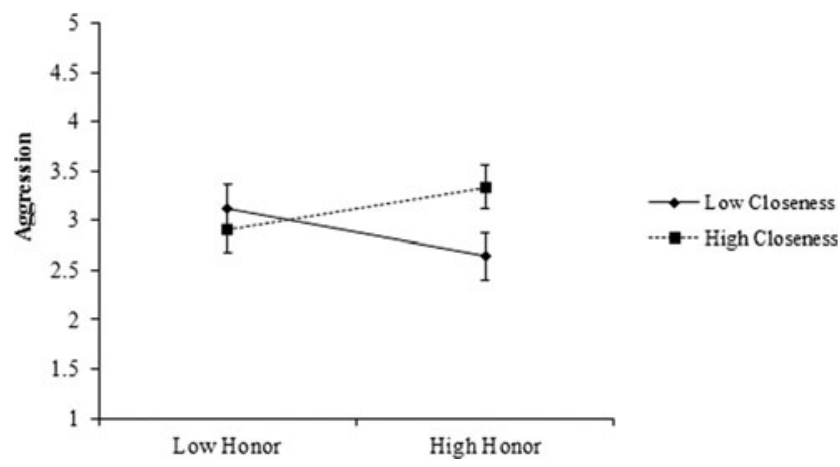

Fig. 1 Study 1 individual honor $\times$ closeness interaction

manipulated, in order to more directly assess whether the mere amount of time a person spends with another is indicative of their likelihood to aggress or whether it is more important that the person feels close to another (without necessarily having to spend extended amounts of time with that person) is indicative of aggression.

\section{Study 2-Laboratory Aggression}

\section{Method}

\section{Participants}

A total of 93 male participants were recruited from a large southwestern university for a laboratory study called "Getting to know others" with a female confederate. Of these, three participants were excluded from analysis: two refused to participate in the experimental task and one knew the confederate. A total of eight participants reported being attracted to men and were removed from analysis. However, note that removing their data did not change the overall results. Thus, the final sample was 82 men $\left(M_{\text {age }}=21.90, S D=6.45\right.$; $75 \%$ Hispanic or Latino, $10 \%$ Caucasian, 8 \% Black, $7 \%$ Asian). Of these, $42 \%$ indicated being in some committed relationship, and $5 \%$ indicated that they were married. Participants were recruited from introductory psychology courses and were compensated with course credit.

\section{Primary Independent Variables}

Honor Like in study 1, we used the revenge subscale of the Culture of Honor Scale (COHS; Figueredo et al. 2004) to assess individual sense of revenge-based honor. The internal consistency was again acceptable (Mean $=-1.27, S D=0.85$, $\alpha=.85$ ).

Bonding Task Participants would be exposed to either a highor low-bonding task with a female confederate. In the highbonding task, participants took turns answering increasingly 
personal questions, whereas participants in the low-bonding task took turns reading a script from one of Shakespeare's lesser-known plays (A Winter's Tale). This task was modeled closely after bonding tasks that have been previously shown to induce romantic attraction (see Aron 2003; Aron et al. 1997). Originally, the control task was going to consist of participants answering non-personal questions, however, Dr. Aron recommended taking turns reading from a script instead because answering non-personal questions still somewhat increases interpersonal closeness (Arthur Aron, November 16, 2012; personal communication). For the high-bonding task, the confederates scripted answers to the bonding questions themselves and their answers were piloted, modified, and rehearsed several times so as to appear fluid, spontaneous, and friendly. The study was launched only after the confederate demonstrated that she had memorized her answers and could repeat them verbatim for each participant. For the low-bonding task, the confederate was instructed to appear generally friendly but to avoid spontaneous personal questions or comments by the participant back to the task.

Interpersonal Closeness The Inclusion of Other in the Self Scale (IOSS; Aron et al. 1992) was also included in this experiment, except it served as a manipulation check for the bonding task.

Value Type We coded participant's self-reported most important value in an identical fashion to study 1.

\section{Dependent Variable}

Aggression We used a proxy measure of aggression by having our participants administer hot sauce to the female confederate after finding out that she strongly dislikes spicy food products (Lieberman et al. 1999). Hot sauce allows the participant to believe that they are causing physical harm to the confederate without them actually being exposed to danger. Thus, the amount of hot sauce the participant gives to the confederate was a rough estimate of aggression toward her. After participants filled a 4-oz paper cup with hot sauce, the cup was weighed using an electronic scale (Mean weight $=6.93, S D=11.20)$. Participants that gave no hot sauce were coded as $1.75 \mathrm{~g}$ (the weight in grams of the empty cup).

\section{Procedure}

Participants entered the lab and were greeted at the door by the experimenter where they were told they would be participating with a partner (the confederate) who had already arrived. Both the participant and the confederate were taken to a large experimental room that was separated into two sections by large opaque dividers separating the participant and confederate. Participants and the confederate were initially separated to fill out preexperimental measures (COHS and demographics). After this, the pair was brought together for the bonding task. To leave the experimenter blind to condition, the participants were randomly handed one of eight sealed envelopes evenly containing either a high- or a low-bonding task. The bonding task ran exactly 15 min, after which the participant and confederate were separated again and would remain separated for the remainder of the study.

Once separated from the confederate, participants filled out two copies of the survey of moral values: one pertaining to the participant's moral values and one pertaining to those of the confederate. Participants were told that the purpose of the experiment was to see if they could predict the confederate's moral values based on a 15-min interaction (the bonding task), and they were told they would be sharing their values with the confederate to see how well they did. These values were identical to study 1 . The participants listed their personal values, predicted the confederate's, and then agreed to let these forms be shared with the confederate. However, in all conditions, the male participant was led to believe that the female participant's values were the exact opposite of his. This represented our moral violation.

Participants then engaged in the aggression task, which they were told was a separate, unrelated study having to do with taste preferences. Participants were asked to record their taste preferences for various foods and drink products such as fruit punch, coffee, hot sauce, and candy. Partners then switched forms, and took note of their partner's food preferences. In all conditions, participants were led to believe that the confederate hates spicy food. The participants were then told that one of them (the "chef") was going to be tasked with administering different foods to the other (the "taster"). In all conditions, the experimenter then pseudo-randomly assigned roles by asking the participant to draw a ballot from a bowl, unaware that all the ballots read "chef." The participant was then given a set of 4-oz paper cups and was asked to fill them with various food items for the confederate to ingest. Participants started with fruit punch, then an extra spicy sample of hot sauce. The hot sauce was a specialty brand that was rated over 350,000 Scoville units of heat (for reference, a jalapeño pepper can range from 1500 to 4500 in heat). Additionally, the hot sauce was diluted with white vinegar to be exceptionally pungent. The participants were encouraged to smell (but not taste) the hot sauce by wafting it toward their nose to confirm that it would be painful to ingest before dispensing it into a cup for the confederate to drink. Participants were again reminded that their partner would have to drink whatever they put in the cup (although no hot sauce was actually ingested). The cup with the hot sauce was then weighed as a behavioral measure of aggression. After participants were done, they were fully debriefed and thanked for their participation. 


\section{Results and Discussion}

Honor and value type were significantly correlated, $r=.22$, $p=.035$, indicating that individuals high in honor were less likely to endorse values that centered on spreading good will or not harming others. Moreover, the manipulation check suggested that the bonding task was successful. Participants reported feeling closer to the confederate in the bonding condition $(M=2.73, S D=1.01)$ than in the control condition $(M=1.74, S D=0.95)$ as measured by the IOSS $(t(81)$ $=4.38, p<.001)$.

\section{Regression Analyses}

An identical regression model to study 1 was used for this study, with the exclusion of relationship length. Thus, we examined the relationship that honor, value type, and closeness had with aggression (as measured by hot sauce) using hierarchical linear regression in step $1\left(R^{2}=.09, F=2.29, p=.086\right)$. Once again, in step $2\left(R^{2}=.14, F=2.27, p=.057\right)$, we included the interaction term of honor $\times$ closeness and honor $\times$ value type. All variables were standardized prior to creating interaction terms. In step 1, there was a significant main effect for individual honor $(\beta=.27 ; 95 \% C I=.04,51 ; p=.023)$, but not for the bonding manipulation $(\beta=.10,95 \% \mathrm{CI}=.14,35$; $p=.390)$ or value type $(\beta=.03,95 \% \mathrm{CI}=-.56, .75$; $p=.770)$. There was no significant interaction between honor and value type, $(\beta=.39,95 \% \mathrm{CI}=-.95,1.71 ; p=.566)$, but as predicted, there was a significant closeness $\times$ honor interaction $(\beta=.24,95 \% C I=.01,47 ; p=.045)$. We graphed this interaction at one standard deviation above and below the mean (see Fig. 2), which revealed that individuals high in individual honor who also bonded with the confederate administered the most hot sauce. This pattern suggested that high individual honor coupled with interpersonal closeness (even when induced in the lab among strangers) increased aggression toward a confederate.

\section{Probing the Interaction}

Simple slopes analyses confirmed that individual honor was significantly related to aggression in the bonding condition $(\beta=.51, p=.002)$ but not in the control condition $(\beta=.08, p=.533)$. To further examine the condition $\times$ honor interaction, we conducted a JohnsonNeyman examination using the SPSS macro "PROCESS" (version 2.12; Hayes 2013). This technique assesses the magnitude by which a participant needed to espouse a $\mathrm{COH}$ to significantly impact their level of aggression in the bond condition (Johnson and Fay 1950; Johnson and Neyman 1936). It revealed that an individual honor score greater than 1.412 standard deviations above the mean was necessary to significantly increase aggression in the bonded group.

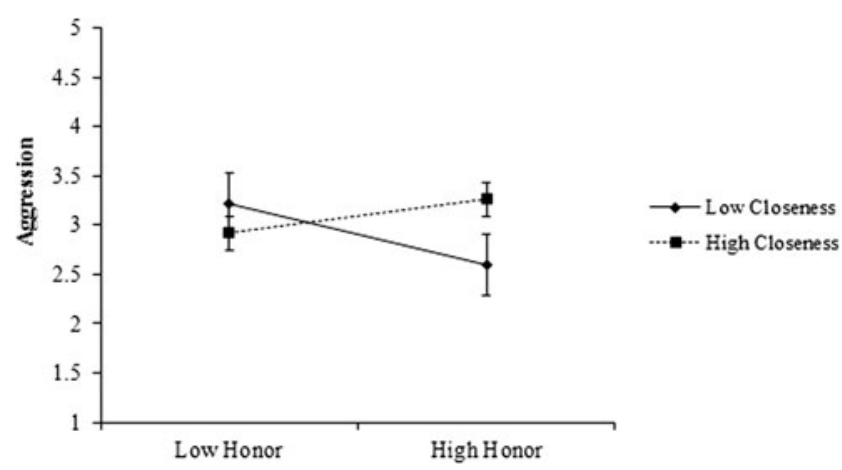

Fig. 2 Study 2 individual honor $\times$ bonding interaction

It should be noted that the participants' relationship status also interacted with honor $(\beta=.28 ; 95 \% C I=.17, .38 ; p<$ $.01)$ and created a significant three-way interaction with honor and closeness $(\beta=.27,95 \% C I=.17, .37 ; p<.01)$. However, the honor $\times$ condition interaction remained significant despite these other predictors $(\beta=.25,95 \% C I=.15$, $36 ; p<.01)$. The pattern of interactions suggests that being in any kind of relationship further increased aggression toward the confederate, which will be discussed in the "General Discussion" section, but was of little theoretical consequence to the interaction of interest. Finally, a post hoc power analysis of this model revealed, with an adjusted observed effect size of 0.1133 , an error probability of 0.05 , and with a total sample size of 90 , this study had a .8027 probability of correctly detecting a significant effect if one exists.

Studies 1 and 2 examined only participants from a $\mathrm{COH}$ (Southwest Texas). It is important to determine whether these results extend to individuals who are not from a $\mathrm{COH}$. Further, the assessment from studies 1 and 2 for individual honor was focused on revenge. Thus, it is unclear whether these results would generalize to other honor assessments. Study 3 was therefore designed to address these limitations.

\section{Study 3-MTurk Replication}

\section{Method}

\section{Participants}

Participants were 280 men recruited from Amazon's MTurk (see Buhrmester et al. 2011; Paolacci et al. 2010) from the USA and were compensated $\$ 0.25$. Our study requested that only men who are currently in relationships take our survey. However, to ensure the veracity of our sample (men who are in relationships), we included the following statement at the end of the survey: "We understand that MTurk is an anonymous place to do tasks. We asked that only men in relationships partake in the survey, but we completely understand that people may have taken the survey who didn't fit that 
description, and that is perfectly OK. Please be honest, we will NOT reject your work, we just need to know for statistical reasons, are you a man who is in a relationship?" Through this procedure, we omitted 39 participants who were either not in a relationship or were not a man. Although this procedure cannot definitively rule out other types of individuals taking our survey, it did seem to be effective in eliminating some. Of the remaining participants, 87 indicated that they were from or lived in a Southern State as defined by Cohen and Nisbett (1994). We focused on these 87 participants (mean age $=34.03, S D=11.86 ; 75 \%$ White, $11 \%$ Black, $8 \%$ Latino/Hispanic $6 \%$ Other).

\section{Design, Measures, and Procedure}

All procedures and measures were similar to study 1 , with minor differences. The first difference is that we used four specific questions (on a $1=$ Strongly disagree to $5=$ Strongly agree Likert scale) to assess aggression: hit her, insult her, punish her, never speak to her again. These items met the threshold for internal consistency $(\alpha=.70)$. A second difference was the inclusion of the Honor Ideology Manhood Scale (or HIM; Barnes et al. 2012a) to assess COH. This 16-item scale has been used in previous research and has excellent validity (Barnes et al. 2012b). This scale was internally consistent in the present sample $(\alpha=.91)$.

\section{Results and Discussion}

None of the independent variables (honor, closeness, and value type) were significantly correlated with each other $(-.20 \leq r \leq .04$, all $p \mathrm{~s}>.05)$. We then ran a hierarchical regression similar to studies 1 and 2 . Honor, closeness, and value type were entered in step 1 and the honor $\times$ closeness and honor $\times$ value type interactions were entered in step 2 . The $R^{2}$ for step 1 was $.11(F=3.37 p=.022)$, and step 2 added significant value $R^{2}=.18(F=3.56, p=.033)$. There was no significant main effect of closeness $(\beta=-.21$; $95 \% \mathrm{CI}=-.44, .02 ; p=.073)$ or value type $(\beta=-.19$; $95 \% \mathrm{CI}=-.44, .06 ; p=.14)$. However, the main effect of honor was significant $(\beta=.37 ; 95 \% \mathrm{CI}=.13, .61 ; p=.003)$. With respect to the interactions, value type $\times$ honor was not significant $(\beta=-.18 ; 95 \% \mathrm{CI}=-.44, .08 ; p=.166)$; however, honor $\times$ closeness was once again significant $(\beta=.29$; $95 \% \mathrm{CI}=.07, .51 ; p=.011)$. Figure 3 shows the pattern of results. Simple slopes analyses show that honor is a significant predictor of aggression at one standard deviation above the mean on closeness $(\beta=.30, p=.017)$, but not one standard deviation below $(\beta=.08, p=.539)$.

It should be noted that the non-southern sample $(n=152)$ had a significant model $\left(R^{2}=.22 ; F=8.21 p<.001\right)$ as well. Value type had no association with aggression $(\beta=0.01$;

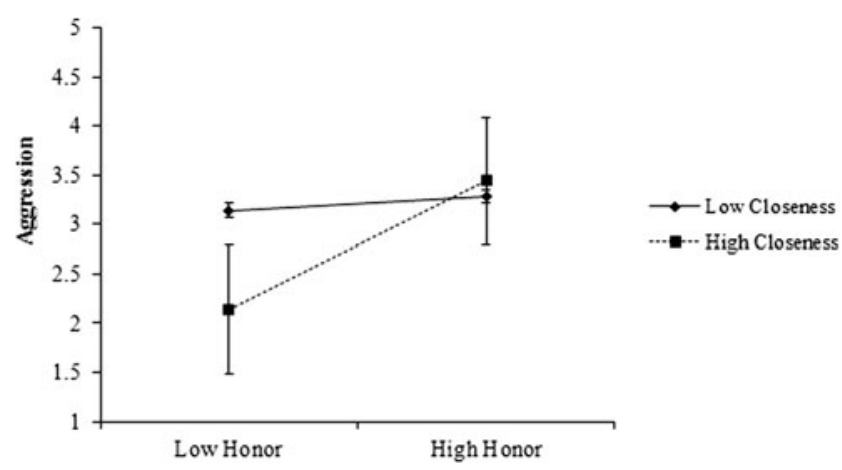

Fig. 3 Study 3 individual honor $\times$ bonding interaction

$95 \% \mathrm{CI}=-.44, .08 ; p=.166)$. However, closeness $(\beta=-.39$; $95 \% \mathrm{CI}=-.54,-.25 ; p<.001)$ was significantly associated with decreased aggression, and honor $(\beta=.22$; $95 \% \mathrm{CI}=-.06, .37 ; p=.007)$ was significantly associated with increased aggression. Although there was no honor $\times$ value type interaction $(\beta=-.06 p=.51895 \% \mathrm{CI}=-.23, .11)$, there was a significant honor $\times$ closeness interaction $(\beta=-.19$ $p=.02995 \% \mathrm{CI}=-.35,-.02)$. Figure 4 shows that honorfocused individuals from non-southern states reported more aggression the more distant they reported being with a partner. Simple slopes analyses shows that honor is significantly associated with aggression at one standard deviation above the mean on closeness $(\beta=.24, p=.002)$, as well as one standard deviation below the mean on closeness $(\beta=.39, p<.001)$.

\section{General Discussion}

Overall, the findings support the idea that men from a $\mathrm{COH}$ who endorse an individual sense of $\mathrm{COH}$, react aggressively to moral violations when interpersonally close to the woman committing the moral violation. Thus, engaging in moral violations, or even merely disagreeing on critical moral values (as in study 2 ), evokes aggression among close others. Our findings are congruent with previous research showing that those highly identified with $\mathrm{COH}$ ideals are more likely to aggress against a female relative over honor-related issues (Cohen et al. 1996).

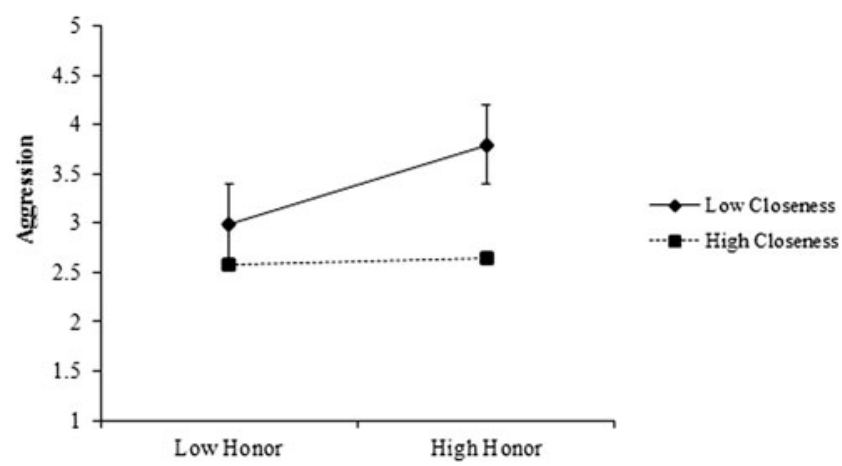

Fig. 4 Study 3 individual honor $\times$ bonding interaction in a non- $\mathrm{COH}$ sample 
However, adding to this literature is the idea that interpersonal closeness increases this aggression, whether that closeness is observed in existing relationships (studies 1 and 3 ) or was experimentally induced (study 2 ). Thus, men who personally endorse high levels of honor will aggress against a woman who does not share his personal moral values, significantly more so if he is close to this woman. However, as study 3 demonstrated, individuals must be from a region where the $\mathrm{COH}$ is endorsed for these effects to emerge. Otherwise, distance from a partner seems to increase aggression from an honor-endorsing man in response to a moral violation.

Interestingly, individuals who were in relationships already were most aggressive toward the confederate in the bonding condition. This finding was not predicted, but perhaps speaks to the efficacy of self-other overlap that occurs during the bonding task. It is critical to note that the female confederate in the present study did not explicitly engage in any moralviolating behaviors. Our confederate merely indicated that she did not value the same moral domain(s) as the participant. Further, it may seem somewhat dubious to argue that a stranger (the confederate) would have an impact on one's selfperceived honor merely through the induction of a laboratory bonding task. However, previous research has used this exact task and found that bonded strangers do become more selflessly altruistic toward each other and these individuals did report an expanded their self-concept (even if only slightly; see Aron et al. 1992). Thus, to the degree that even a small expansion of the self occurs during a bonding task (albeit temporarily), it follows - and this study demonstrates - that a threat to one's sense of honor is possible from even a 15min bonding interaction.

Our participants for studies 1 and 2 were collected from a region of the USA (Southwest Texas) that is high in honor culture (Cohen and Nisbett 1994). Thus, these findings should not generalize to non-honor-related areas (e.g., Maine). Indeed, study 3 demonstrated that these processes are not the same for those who do not live or come from honor-based regions of the USA. In fact, study 3 suggests that, honorendorsing individuals outside of a $\mathrm{COH}$ exhibit a negative association between closeness and aggression against a moral-violating romantic partner. One strength of the present research is that we sampled separate populations (students and Mechanical Turk workers), which provides increased external validity (Buhrmester et al. 2011; Paolacci et al. 2010). Further, we argue that these findings would translate well to other populations that endorse honor-based values (see Figueredo et al. 2004).

In sum, these studies suggest that an individual sense of honor contributes to aggression against perceived moral violations, and those aggressive acts are exacerbated by interpersonal closeness. These findings build on previous research linking family honor and aggression and provide an additional piece of the puzzle in understanding the baffling crimes characterized by honor-related violence. Counterintuitively, but aligned with the evolutionary literature, it appears that the closer a high $\mathrm{COH}$ man is to a woman, the greater risk she may have for being the victim of honor-related violence. These findings further suggest that the men who commit these crimes are not callous, but deeply connected with their belief system and interpersonally close to the women who endure these attacks. Further, morality appears to be a provoking situation that may have been faced in ancestral times that elicited close-other aggression.

Acknowledgments We would like to thank our research assistants, Alejandra Sanchez and Jasmine Lopez, for their work as confederates. We would also like to thank the Society for the Psychological Study of Social Issues (SPSSI) for a grant-in-aid related to this project.

\section{References}

Anderson, K. L. (2002). Perpetrator or victim? Relationships between intimate partner violence and well-being. Journal of Marriage and Family, 64, 851-863.

Aron, A. (2003). Self and close relationships. In M. Leary \& J. Tangney (Eds.), Handbook of self and identity (pp. 442-461). New York: The Guilford Press.

Aron, A., \& Aron, E. N. (1986). Love and the expansion of self: understanding attraction and satisfaction. New York: Hemisphere Publishing Corp/Harper \& Row Publishers.

Aron, A., Aron, E. N., \& Smollan, D. (1992). Inclusion of Other in the Self scale and the structure of interpersonal closeness. Journal of Personality and Social Psychology, 63, 596-612.

Aron, A., Mellinat, E., Aron, E. N., Vallone, R. D., \& Bator, R. J. (1997). The experimental generation of interpersonal closeness: a procedure and some preliminary findings. Personality and Social Psychology Bulletin, 23, 363-377.

Barnes, C. D., Brown, R. P., \& Osterman, L. L. (2012a). Don't tread on me masculine honor ideology in the US and militant responses to terrorism. Personality and Social Psychology Bulletin, 38, 10181029.

Barnes, C. D., Brown, R. P., \& Tamborski, M. (2012b). Living dangerously culture of honor, risk-taking, and the nonrandomness of "accidental" deaths. Social Psychological and Personality Science, 3, $100-107$.

Baumeister, R. F., Smart, L., \& Boden, J. M. (1996). Relation of threatened egotism to violence and aggression: the dark side of high selfesteem. Psychological Review, 103(1), 5-33.

Baumeister, R. F., Vohs, K. D., \& Funder, D. C. (2007). Psychology as the science of self-reports and finger movements: whatever happened to actual behavior? Perspectives on Psychological Science, 2, 396403.

Buhrmester, M., Kwang, T., \& Gosling, S. D. (2011). Amazon's Mechanical Turk a new source of inexpensive, yet high-quality, data? Perspectives on Psychological Science, 6, 3-5.

Buss, D. M., \& Duntley, J. D. (2006). The evolution of aggression. In M. Schaller, J.A. Simpson, \& D. T. Kenrick (Eds.), Evolution and social psychology, pp. 263-286.

Chesler, P. (2009). Are honor killings simply domestic violence? Middle East Quarterly, 16, 61-69.

Cohen, D., \& Nisbett, R. E. (1994). Self-protection and the culture of honor: Explaining southern violence. Personality and Social Psychology Bulletin, 20, 551-567. 
Cohen, D., Nisbett, R., Bowdle, B., \& Schwarz, N. (1996). Insult, aggression, and the southern culture of honor: an "experimental ethnography.". Journal of Personality and Social Psychology, 70, 945-960.

Daly, M., \& Wilson, M. (1988). Homicide. Brunswick: Transaction Publishers.

Daly, M., Wilson, M., \& Weghorst, S. J. (1982). Male sexual jealousy. Ethology and Sociobiology, 3(1), 11-27.

Dawkins, R. (2006). The selfish gene (No. 199). Oxford: Oxford University press.

Figueredo, A., Tal, I., McNeil, P., \& Guillén, A. (2004). Farmers, herders, and fishers: the ecology of revenge. Evolution and Human Behavior, 25, 336-353. doi:10.1016/j.evolhumbehav.2004.05.005.

Graham, J., Haidt, J., \& Nosek, B. A. (2009). Liberals and conservatives rely on different sets of moral foundations. Journal of Personality and Social Psychology, 96(5), 1029.

Haidt, J. (2008). Morality. Perspectives on Psychological Science, 3, 6572.

Hayes, A. F. (2013). Introduction to mediation, moderation, and conditional process analysis: a regression-based approach. New York: The Guilford Press.

Henrich, J. (2009). The evolution of costly displays, cooperation and religion: credibility enhancing displays and their implications for cultural evolution. Evolution and Human Behavior, 30, 244-260.

Johnson, P. O., \& Fay, L. C. (1950). The Johnson-Neyman technique, its theory and application. Psychometrika, 15, 349-367.

Johnson, P. O., \& Neyman, J. (1936). Tests of certain linear hypotheses and their application to some educational problems. Statistical Research Memoirs, 1, 57-93.

Leung, A. K., \& Cohen, D. (2011). Within- and between-culture variation: Individual differences and the cultural logistics of honor, face, and dignity cultures. Journal of Personality and Social Psychology, 100, 507-526. doi:10.1037/a0022151.
Lieberman, J. D., Solomon, S., Greenberg, J., \& McGregor, H. A. (1999). A hot new way to measure aggression: hot sauce allocation. Aggressive Behavior, 25, 331-348.

Nisbett, R. E. (1993). Violence and US regional culture. American Psychologist, 48, 441-449.

Paolacci, G., Chandler, J., \& Ipeirotis, P. G. (2010). Running experiments on Amazon Mechanical Turk. Judgment and Decision Making, 5, 411-419.

Robins, R. W., Hendin, H. M., \& Trzesniewski, K. H. (2001). Measuring global self-esteem: construct validation of a single-item measure and the Rosenberg self-esteem scale. Personality and Social Psychology Bulletin, 27, 151-161.

Rosenberg, M. (1965). Society and the adolescent self-image (p. 326). Princeton: Princeton University Press.

Ross, P. (2013). Pakistani girl axed 15 times: why did brother of gul meena attempt 'honor killing'? International Science Times, Retrieved from http://www.isciencetimes.com/articles/4863/ 20130405/pakistan-honor-killing-gul-meena-axed-afghanistan.htm.

Salmivalli, C. (2001). Feeling good about oneself, being bad to others? Remarks on self-esteem, hostility, and aggressive behavior. Aggression and Violent Behavior, 6, 375-393.

Sev'er, A., \& Yurdakul, G. (2001). Culture of honor, culture of change: a feminist analysis of honor killings in rural Turkey. Violence Against Women, 7, 966-1000.

Sommers, T. (2009). The two faces of revenge: moral responsibility and the culture of honor. Biological Philosophy, 24, 35-50.

Tabak, B. A., McCullough, M. E., Szeto, A., Mendez, A. J., \& McCabe, P. M. (2011). Oxytocin indexes relational distress following interpersonal harms in women. Psychoneuroendocrinology, 36, 115122.

Vandello, J. A., \& Cohen, D. (2003). Male honor and female fidelity: implicit cultural scripts that perpetuate domestic violence. Journal of Personality and Social Psychology, 84, 997-1010. 